\title{
Modeling oxidative injury response in human kidney organoids
}

\author{
Aneta Przepiorski ${ }^{1}$, Thitinee Vanichapol ${ }^{2}$, Eugenel B. Espiritu ${ }^{1}$, Amanda E. Crunk ${ }^{1}$, Emily Parasky ${ }^{1}$, \\ Michael D. McDaniels ${ }^{1}$, Dave R. Emlet ${ }^{3}$, Ryan Salisbury ${ }^{4}$, Cassandra L. Happ ${ }^{4}$, Lawrence A. Vernetti ${ }^{5}$, \\ Matthew L. MacDonald ${ }^{4}$, John A. Kellum³, Thomas R. Kleyman ${ }^{6}$, Catherine J. Baty ${ }^{6}$, Alan J. Davidson² and \\ Neil A. Hukriede ${ }^{1 *}$ (1)
}

\begin{abstract}
Background: Hemolysis occurs in many injury settings and can trigger disease processes. In the kidney, extracellular hemoglobin can induce damage via several mechanisms. These include oxidative stress, mitochondrial dysfunction, and inflammation, which promote fibrosis and chronic kidney disease. Understanding the pathophysiology of these injury pathways offers opportunities to develop new therapeutic strategies.

Methods: To model hemolysis-induced kidney injury, human kidney organoids were treated with hemin, an ironcontaining porphyrin, that generates reactive oxygen species. In addition, we developed an induced pluripotent stem cell line expressing the biosensor, CytochromeC-GFP (CytoC-GFP), which provides a real-time readout of mitochondrial morphology, health, and early apoptotic events.

Results: We found that hemin-treated kidney organoids show oxidative damage, increased expression of injury markers, impaired functionality of organic anion and cation transport and undergo fibrosis. Injury could be detected in live CytoC-GFP organoids by cytoplasmic localization of fluorescence. Finally, we show that 4-(phenylthio)butanoic acid, an HDAC inhibitor with anti-fibrotic effects in vivo, reduces hemin-induced human kidney organoid fibrosis.

Conclusion: This work establishes a hemin-induced model of kidney organoid injury. This platform provides a new tool to study the injury and repair response pathways in human kidney tissue and will assist in the development of new therapeutics.
\end{abstract}

Keywords: Kidney organoids, iPSCs, Renal injury, ROS, Hemin, Mitochondria, Cytochrome C

\section{Background}

Intravascular hemolysis occurs in many disease settings such as rhabdomyolysis, sepsis, ischemia reperfusion injury (IRI), sickle cell anemia or cardiac bypass $[1,2]$. Hemolytic events can lead to acute injury events, and recurrent hemolysis can eventually lead to chronic kidney disease (CKD). Under healthy conditions,

\footnotetext{
*Correspondence: hukriede@pitt.edu

${ }^{1}$ Department of Developmental Biology, University of Pittsburgh, School of Medicine, 3501 5th Ave., 5061 BST3, Pittsburgh, PA 15213, USA

Full list of author information is available at the end of the article
}

cell-free hemoglobin is present in circulation at very small amounts due to scavenging proteins such as haptoglobin or hemopexin [3]. Under pathological conditions, when hemolysis occurs, cell-free hemoglobin is released in significant amounts into the circulation [4]. The sudden release of cell-free hemoglobin, also releasing heme and iron, can overwhelm the mechanisms in place that neutralize the impact, and as a result, lead to cellular injury. Iron levels are also tightly regulated in the body and are normally supplied via absorption in the gut or recycling of senescent red blood cells. Under healthy conditions ferrous iron $\left(\mathrm{Fe}^{2+}\right)$ is bound with heme. However, original author(s) and the source, provide a link to the Creative Commons licence, and indicate if changes were made. The images or other third party material in this article are included in the article's Creative Commons licence, unless indicated otherwise in a credit line to the material. If material is not included in the article's Creative Commons licence and your intended use is not permitted by statutory regulation or exceeds the permitted use, you will need to obtain permission directly from the copyright holder. To view a copy of this licence, visit http://creativecommons.org/licenses/by/4.0/. The Creative Commons Public Domain Dedication waiver (http://creativeco mmons.org/publicdomain/zero/1.0/) applies to the data made available in this article, unless otherwise stated in a credit line to the data. 
if it becomes released into the circulation, it is very quickly oxidized to $\mathrm{Fe}^{3+}$ and $\mathrm{Fe}^{4+}$. Cell-free hemoglobin and heme are highly reactive and will facilitate formation of other reactive oxygen, nitrogen, and lipid molecules. Hemin is the $\mathrm{Fe}^{3+}$ oxidized form of heme which under serum or protein-free conditions can be used in cell culture to study cell responses [5].

Renal injury, due to hemoglobin and heme, appears to be multifactorial including cellular membrane damage, oxidative stress imbalance, reaction with other proteins and lipids, and activation of immune responses. Due the hydrophobic nature of heme, it readily binds to cellular and intracellular membranes and proteins leading to lipid peroxidation and altered protein function [6-8]. Once heme is internalized by the cells, it is catabolized by heme oxygenase 1 (HMOX1) enzyme. This reaction is rate limiting and the breakdown of heme produces biliverdin, iron, and carbon monoxide, which are released into the cytosol [9]. Even though carbon monoxide and biliverdin have anti-inflammatory effects, release of iron can trigger Fenton reactions, which generates increases in hydroxyl radicals and oxidative stress that can overwhelm the cellular antioxidant capacity leading to injury $[6,10]$.

Reactive oxygen species (ROS) are generated as part of normal metabolic functions [11]. Mitochondria are the most metabolically active organelles in the cell and generate the greatest amounts of ROS, releasing most of it in the form of hydroxyl. Since cell-free heme and iron are highly reactive with hydroxyl radicals, mitochondria are vulnerable to injury. Studies conducted in rats using glycerol to cause rhabdomyolysis induced acute kidney injury (AKI), show that mitochondria readily accumulate heme within hours of injury, leading to a rapid rise in oxygen consumption and corresponding increase in ROS. This immediate effect is followed by a reduction in both oxygen consumption and transmembrane potential leading to mitochondrial dysfunction [12]. Another consequence of increased ROS is reaction of hydroxyl radicals with nitric oxide (NO) to form peroxynitrite. Peroxynitrite modifies tyrosine residues on proteins to 3-nitrotyrosine [13], which leads to altered protein and enzymatic function, lipid peroxidation, and cellular damage [14]. To study ROS-mediated injury in real-time, we developed a novel biosensor induced pluripotent stem cells (iPSC) line, using CytochromeC-GFP (CytoC-GFP) [15]. CytoC is a transmembrane protein that is localized to the inner mitochondrial membrane and is part of the electron transport chain. In healthy cells CytoC acts as an electron carrier to reduce the levels of ROS produced by the mitochondria [16]. However, rapid increases in ROS (such as found in cellular injury) results in CytoC translocating to the cytoplasm where it activates the caspase cascade in the apoptotic pathway [17].
Developing targeted renal therapeutics has resulted in no approved drugs, to date. While there are several possible reasons for this, one possibility is that results from preclinical murine models do not consistently translate to human studies [18]. One way to address this shortcoming is to utilize human kidney organoids. Many protocols have now been developed to generate kidney organoids to study kidney injury and disease pathways in vitro. However, few models of injury have been developed, and to date, most have focused on nephrotoxin (cisplatin, gentamicin) mediated injury [19-25]. Using a simple bioreactor-based method for generating human kidney organoids from iPSCs $[26,27]$, we hypothesized hemin-induced ROS-mediated injury and fibrosis could be a valuable tool for evaluating potential therapeutics. Here, we show how this model can be used to test the efficacy of promising anti-fibrotic agents such as 4-(phenylthio)butanoic acid (PTBA), which we have previously shown in mouse models can ameliorate injury and fibrosis [28-32].

In this study, we developed a human kidney organoid hemolytic injury model that can be utilized to study acute injury, mitochondrial dysfunction, and fibrosis. We chose to use hemin to induce injury, as hemin is a potent ROS inducer, a central player in renal damage, and mimics the hemolytic response seen in many diseases leading to renal injury [3]. As such, we hypothesize this model can be used to extrapolate common disease pathways and focus on therapeutic outcomes that will be widely applicable a for multi-factorial renal disease.

\section{Methods \\ iPSC culture}

All work was performed in compliance with institutional guidelines (IBC201600244), and was carried out in a Class II biosafety hood with appropriate personal protective equipment. iPSCs were maintained on $10 \mathrm{~cm}$ cell culture dishes coated with Geltrex (Thermo Fisher) or Cultrex (R\&D) and mTeSR1 (Stemcell Technologies) medium, passaged every 3-4 days. Experiments were performed with MANZ-2-2 (female) and MANZ4-37 (male) iPSC lines [33] as stated in the figures.

\section{Kidney organoid assays and multi-well magnetic spinner} Kidney organoid assays and setting up of magnetic stirplate were performed as described previously with modifications to the original Przepriorski et al. 2018 protocol [27].

\section{Hemin treatment}

Hemin (Millipore-Sigma) $10 \mathrm{mM}$ stock concentration was resuspended in $0.1 \mathrm{M} \mathrm{NaOH}$, sterile filtered and prepared fresh for every experiment. Day 14 organoids were 
washed thrice with DMEM-low glucose, then transferred into protein-free medium (1:1 ratio of DMEM-low glucose and Hams F-12 Nutrient mixture, $1 \times$ HEPES (to stabilize $\mathrm{pH}$ ) $1 \%$ penicillin/streptomycin (Gibco), and $2.5 \mathrm{ug} / \mathrm{mL}$ Plasmocin) containing hemin in a 6-well ultralow attachment (ULA) plate. The assay was then placed on a magnetic stir plate (2mag-USA) at $120 \mathrm{rpm}, 25 \%$ power. Except where stated, hemin concentration was at $25 \mu \mathrm{M}$. Control well contained equivalent volume of $0.1 \mathrm{M} \mathrm{NaOH}$ as a vehicle control. All treatments were maintained for $48 \mathrm{~h}$, and thereafter washed thrice with Stage II medium (DMEM-low glucose, 10\% knock-out serum replacement, $1 \%$ penicillin/streptomycin (Gibco), 1\% Glutamax (Gibco), 1\% HEPES, 1\% MEM non-essential amino acids, $0.5 \%$ polyvinyl alcohol, $2.5 \mathrm{ug} / \mathrm{mL}$ Plasmocin) before proceeding to compound treatment. The $\mathrm{pH}$ of the control and hemin-containing media was tested following $48 \mathrm{~h}$ incubation to exclude possibility of injury due to $\mathrm{pH}$ changes. The $\mathrm{pH}$ tested was 7.7 for control and 7.55 for hemin, within the normal range shown not to affect cellular apoptosis [34].

\section{Compound treatments}

Day 16 kidney organoids (post hemin treatment) were treated daily with UPHD25 compound [30, 31]. Stage II medium supplemented with 0.3\% DMSO (Stage IIDMSO) was prepared. Firstly, a $2 \times$ stock solution of compound was prepared in Stage II-DMSO, and a calculated amount was added to each well to make up $1 \times$ working solution in a total of $3 \mathrm{~mL}$ volume, per well of a 6-well ULA plate. The plates were maintained on the magnetic stirrer at $25 \%$ power and 120 revolutions until fixation at day 26 .

\section{RNA extraction, CDNA synthesis and QPCR}

Organoids were washed in PBS and homogenized in TRIzol (Thermo Fisher). Total RNA was extracted first using Phase Separation Reagent (Molecular Research Center) and using Qiagen RNeasy kit. cDNA was synthesized using qScript cDNA SuperMix (Quanta). qPCR was performed using the Power SYBR Green reagent (Thermo Fisher) on a QuantStudio 12 Flex Real-Time PCR machine. Gene expression was calculated using the $\mathrm{dCt}$ method using HPRT1 for normalization [35]. Error bars represent standard deviation of triplicate measurements. All qPCR analyses were performed in organoids derived from three independent kidney organoid assays and representative results are shown.

\section{RNA-Seq and analysis}

Total RNA from quadruplicate samples of control and hemin treated MANZ2-2 kidney organoids ( $\sim 100$ organoids/sample) was prepared using TRIzol and Phase
Separation Reagent, purified using Ambion PureLink RNA Mini Kit with in-column RNase-Free DNase I (Qiagen) treatment. All samples contained $>1 \mu \mathrm{g}$ total RNA. All samples sequenced had a RIN value of $\geq 9.4$. Library preparation was done using TruSeq Stranded mRNA $($ PolyA +$)$ kit, and sequencing on Illumina Sequencing using NextSeq500. Quality control, library preparation and sequencing were performed by Health Sciences Sequencing Core, UPMC Children's Hospital of Pittsburgh. Reads were mapped on the human genome, GRCh38.p13 using STAR [36] and counted using featureCounts [37]. Differential expression analysis was carried out using edgeR [38] and limma [39] with a threshold of $\log$ fold change $=1$ and adjusted $\mathrm{P}$ value of $<0.05$. Gene set enrichment analysis (GSEA) was performed for the hallmark gene sets and Gene Ontology (GO) classification from the MSigDB collections using the clusterProlifer package [40-43]. The RNA sequencing (RNA-Seq) data were deposited in the National Center for Biotechnology Information's GEO database (GSE182350).

\section{CytoC-GFP iPSC line development and imaging}

CytoC-GFP iPSC lines were generated using AAVS1 Safe Harbor Site Targeting 2.0 Complete Kit 2.0, with an allpurpose HR donor vector (System Biosciences). The CytoC-GFP construct generated in Douglas Green's lab (Addgene \#41,182) [15] was cloned into the AAVS1-SApuro-EF1 $\alpha-M C S$ donor vector using Cold Fusion Cloning kit (System Biosciences). The donor vector was transfected into the MANZ2-2 cell line using Lipofectamine stem reagent according to manufacturer's specifications (ThermoFisher). When the plated cells reached 70\% confluency puromycin was added at $0.5 \mu \mathrm{g} / \mathrm{mL}$ to select positive cells that underwent homologous recombination and integration of the donor construct. After 10 days of daily treatment with puromycin, stable colonies positive for EF1A-CytoC-GFP emerged. Colonies which had the strongest and most homogeneous expression of CytoCGFP in the mitochondria were manually isolated and expanded to generate individual cell lines. Three cell lines were selected for further validation and experiments. Live organoids treated with hemin were imaged on a Leica Sp8 confocal in coverglass bottom $30 \mathrm{~mm}$ dish (Cellvis) in protein free medium using an OKO environmental chamber at $40 \times$ water objective $1.1 \mathrm{NA}, 0.42 \mu \mathrm{m}$ step, $3 z$.

\section{Live MitoTracker Red CMXRos staining}

Stock concentration of $1 \mathrm{mM}$ MitoTracker Red CMXRos was prepared in DMSO according to manufacturer's instructions. CytoC-GFP iPSC lines and organoids were washed with DPBS, and then incubated for $30 \mathrm{~min}$ in a working solution of $500 \mathrm{nM}$ MitoTracker Red CMXRos 
in mTeSR1 or Stage 2 medium respectively. Kidney organoids were incubated for at least $1 \mathrm{~h}$ before imaging. Cell were then washed in DPBS and imaged in FluoroBrite $^{\text {TM }}$ DMEM (Gibco) on the Zeiss LSM700 confocal microscope.

\section{Live dihydroethidium (DHE) staining}

Stock concentration of $5 \mathrm{mM}$ DHE was prepared in DMSO according to manufacturer's instructions. CytoCGFP iPSC organoids were washed with DPBS, and then incubated for $1 \mathrm{~h}$ in a working solution of $5 \mu \mathrm{M}$ DHE in stage 2 medium. Organoids were then washed in DPBS and imaged in FluoroBrite ${ }^{\mathrm{TM}}$ DMEM (Gibco) on the Zeiss LSM700 confocal microscope.

\section{Methods of functional transport assay}

Organoids were loaded in $1 \mathrm{mM}$ 6-carboxyfluorescein (6CF) diluted in OptiMem for $35 \mathrm{~min}$ at $37^{\circ} \mathrm{C}$ and then $10 \mathrm{mM}$ of ethidium bromide was added to mixture for remaining $10 \mathrm{~min}$. Organoids were then washed prior to imaging in OptiMem in a $30 \mathrm{~mm}$ coverglass bottom dish. A Leica SP8 confocal microscope with Okolab environmental chamber $\left(37{ }^{\circ} \mathrm{C}\right.$ and $\left.5 \% \mathrm{CO}_{2}\right)$, motorized stage, and $25 \times 0.95 \mathrm{~N}$. A water immersion objective was used for live cell imaging. Conventional settings for fluorescein (488 $\mathrm{nm}$ laser line) and ethidium bromide (552 laser line) were used with sequential between line acquisition to avoid bleed through, bidirectional imaging for speed and $2 \mathrm{~mm}$ step size. At least ten well organized kidney organoids were imaged for each treatment group. Tubular structures were scored based on maximum projection views, cut through midsection from three different experiments. Experiments were carried out with MANZ2-2 iPSC line.

\section{Histochemistry and analysis}

Kidney organoids fixed in $4 \%$ paraformaldehyde and embedded in paraffin as previously described [26]. Briefly, $6 \mu \mathrm{m}$ thick sections were deparaffinized and heat-induced antigen retrieval performed using sodium citrate $\mathrm{pH} 6.0$ buffer. Primary antibodies used were as follows; HAVCR1/KIM-1 (R\&D Systems, AF1750), $\gamma \mathrm{H} 2 \mathrm{AX}$ (ThermoFisher, 50-194-123), HMOX-1 (Santa Cruz, sc-136960), nitrotyrosine (Novus, NB110-96877), Collagen 1a1 (Abcam, ab138492), Nephrin (PROGEN Biotechnik, GP-N2) CDH1 (BD Biosciences, 610182), Casp3 (BD Biosciences, 559565), KI67 (GenTex, GTX16667), MEIS1/2/3 (Antibodies-online, ABIN2668724). Collagen hybridizing peptide (CHP-Cy3, 3Helix) staining was performed after deparaffinization, according to manufacturer's instructions. Fluorescently stained sections were imaged on a Zeiss LSM700 confocal microscope. All CHP and COL1A1 imaging was done under the same settings established on the no-hemin control. Analysis was performed using ImageJ by combining all of the single channel images into one stack, subtracting the background (rolling ball radius of 50.0 pixels, sliding parapoloid), and applying a threshold. Threshold was determined based on the controls for each assay, and subsequently applied to the stack. Area of the threshold was then measured and calculated by division of the DAPI threshold area value. For analysis at least 3 assays were examined with $>10$ individual organoid sections per condition.

\section{Proteomic analysis}

Approximately 100 organoids per sample $(25 \mu \mathrm{g}$ of 100 $\mu \mathrm{g}$ of total protein used per sample) were washed in cold PBS three times and flash frozen $-80{ }^{\circ} \mathrm{C}$ until processing. Samples were thawed on ice and resuspended in 1X SDS solubilization buffer (5\% SDS in $50 \mathrm{mM}$ TEAB), reduced by the addition of dithiothreitol (DTT) at a final concentration of $20 \mathrm{mM}$ and heated to $950 \mathrm{c}$ for $10 \mathrm{~min}$. Alkylation was performed by the addition of iodoacetamide (IAA) to a final concentration of $40 \mathrm{mM}$ followed by incubation in the dark for $30 \mathrm{~min}$ at room temperature. The SDS lysate was acidified with $12 \%$ aqueous phosphoric acid, 1:10 (v/v) to a final concentration of $1.2 \%$. The solution was then diluted with the S-Trap protein binding buffer (90\% methanol, $0.1 \mathrm{M} \mathrm{TEAB}, \mathrm{pH}$ 7.55), 6:1 (v/v, S-Trap: total vol.). The mixture was transferred to the S-Trap ${ }^{\text {TM }}$ micro spin column, centrifuged at $4,000 \mathrm{~g}$ for $20 \mathrm{~s}$ and processed according to manufacturer's instructions. The three eluents were pooled and dried with vacuum centrifuging at $4{ }^{\circ} \mathrm{C}$ (Labconco, Kansas City, MO, USA). Peptides were re-suspended with $20 \mu \mathrm{L}$ of $0.1 \%$ formic acid (FA) for fractionation. Fractionation was performed as described in the manufacturer's manual (Cat No. 84868, Thermo Fisher Scientific). Desalted peptides were reconstituted in $0.1 \%$ FA and peptide concentration was determined using the Pierce ${ }^{\mathrm{TM}}$ BCA Protein Assay Kit. Samples were normalized to $0.5 \mu \mathrm{g} / \mu \mathrm{L}$. Peptides were TMT-labeled as described previously [44]. TMT-tagged peptides were diluted to $300 \mu \mathrm{L}$ with $0.1 \%$ trifluoroacetic acid. The two sets of 10 plex TMT tags were used to label peptides from a total of 18 samples. The samples were placed into separate batches with each experimental group $(n=3)$ represented in each batch. After quantification of the tagged peptides a pooled internal reference standard for each batch was made using equal volumes of each sample. The internal reference standard was used as the basis for determining the relative abundance of each experimental sample across the two different batches. The samples were run on a Thermo Scientific $^{\mathrm{TM}}$ Orbitrap $^{\mathrm{TM}}$ Tribrid $^{\mathrm{TM}}$ mass spectrometer and analyzed with Proteome Discoverer ${ }^{\mathrm{TM}}$ Software. Experiment was carried out with MANZ2-2 iPSC line. 


\section{Statistical analysis}

Statistical significance was determined using one-way ANOVA, unpaired $t$ with Welch's correction in Prism (GraphPad). $P$ values of $<0.05$ were considered to be statistically significant.

\section{Results}

Hemin-induced injury in kidney organoids models kidney injury

Based on a previous studies, on which our organoid induction method is modeled, we hypothesized day 14 would be ideal for initiating hemin treatments, as the organoids are well formed at this stage and proliferation required to establish the nephrons has begun to diminish [26]. As the previously published protocol [26] was recently modified [27], we first confirmed that all the renal segment markers are expressed. We found that organoids contained the segmentation pattern as shown in previous protocols, with LTL and CDH1 labelling proximal and distal tubule respectively, NPHS1 labelling podocyte clusters and MEIS1/2/3 confirming presence of interstitial cells (Additional file 1: Fig. S1). Additionally, day 14 kidney organoids had low level of cells undergoing double-stranded breaks $(\gamma \mathrm{H} 2 \mathrm{AX})$ and a proportion of the MEIS1/2/3 positive interstitial cells and tubules were proliferating (Additional file 1: Fig. S1). Since the day 14 organoids expressed the expected segment markers, we wanted to confirm if heme binding, and iron transporters were present to assay functional outcomes of hemin injured kidney organoids, therefore we performed both proteomic and RNA-Seq studies. We found day 14 kidney organoids expressed many heme pathway proteins necessary for homeostasis and hemolytic injury, including heme binding proteins (HEBP1, HEBP2), enzymes involved in catabolism of heme (BLVRA, BLVRB), iron transport (ACO1, LRP1, TFRC) and heme biosynthesis (ALAD, HMBS, UROD) (Additional file 1: Fig. S2). We also examined lower abundance, membrane-bound heme and iron transporters required for cellular heme and iron handling by RNA-Seq, and found expression of $L R P 2$, SLC40A1, HRG, SLC39A14, SLC11A2, SLC39A8 at day 14 (Additional file 1: Fig. S3) [45-47].

Previous studies in cell culture monolayers have reported hemin dose treatments ranging between 10 and $30 \mu \mathrm{M}$ lead to an increase in apoptosis and oxidative stress $[48,49]$. To investigate whether hemin could be used to reproducibly induce injury in kidney organoids and recapitulate hemolytic events, we tested a range of hemin concentrations. Day 14 kidney organoids were treated with $6.25 \mu \mathrm{M}$ to $100 \mu \mathrm{M}$ hemin in a proteinfree medium for $48 \mathrm{~h}$. Following treatment, the extent of injury was examined by quantitative PCR for key inflammatory markers known to be upregulated during heme induced injury [50]. Induction of $H M O X 1$, an enzyme that catabolizes hemin and is induced by ROS, showed a concentration-dependent and transient increase in gene expression (Fig. 1a; Additional file 1: Fig. S4A, B) indicative of a successful response to hemin treatment [51]. Expression of HAVCR1 (a kidney injury marker, also known as KIM1) increased more than threefold with $25 \mu \mathrm{M}$ of hemin, and more than a sixfold in concentrations of $50 \mu \mathrm{M}$ and above, suggesting that proximal tubules in the kidney organoids were injured (Fig. 1a). A similar response was observed with the inflammatory markers IL6 (17.4 fold at $25 \mu \mathrm{M}, 113.3$ fold at $50 \mu \mathrm{M}$, and 350 fold at $100 \mu \mathrm{M}$ ) and CXCL8 (5.7 fold at $25 \mu \mathrm{M}, 23.2$ fold at $50 \mu \mathrm{M}$, and 51 fold at $100 \mu \mathrm{M})$. Based on these results we selected the $25 \mu \mathrm{M}$ dose for further characterization as this was the lowest dose where a reproducible increase in all the injury/inflammatory markers was observed.

We next performed a time-course analysis for HMOX1, HAVCR1, IL6 and CXCL8 from day 15 (24 h post hemin addition) to day 20 of organoid culture (Fig. 1b; Additional file 1: Fig. S4C, D). Consistent with HMOX1 being induced by ROS, its expression is maximal at day 15 (143.6 fold over control), and then sharply drops off once hemin is removed at day 16 (37.5 fold on day 16 and then low levels until day 20; Fig. 1b). This profile is roughly paralleled by IL6 and CXCL8 with a more gradual decline to day 20 (Fig. 1b; Additional file 1: Fig. S4C, D). Expression of HAVCR1 shows a more complex profile with peaks of high expression relative to controls at day 15 and 20 (Fig. 1b). Immunofluorescence staining at these stages, as well as day 26 , showed that hemin-treated organoids display high levels of HAVCR1 in the proximal tubules (labelled by Lotus tetragonolobus lectin; LTL) compared to the

(See figure on next page.)

Fig. 1 Hemin injury leads to induction of inflammation and nitric oxide mediated nitrosylation. a Representative quantitative PCR (qPCR) of kidney organoids treated with hemin at increasing concentrations. Expression relative to the control (untreated) organoids. b Time course qPCR of control and hemin treated organoids from day 15 to day 20. Results show mean and standard deviation of 3 technical replicates. c Immunofluorescence of kidney organoid paraffin sections of control and hemin treated organoids, showing localization of kidney injury marker 1 (KIM1, red) to proximal tubules labelled with Lotus tetragonolobus lectin (LTL, green), apoptotic marker activated caspase 3 (Casp3, red), DNA damage marker ( $\gamma H 2 \mathrm{AX}$, green), heme catabolizing enzyme heme oxygenase 1 (HMOX1, orange), and nephrin labelling podocytes (NPHS1, red). d Immunofluorescence of kidney organoid paraffin sections at day 16, 18 and 20 showing 3-nitrotyrosine staining (red) indicative of nitrositive stress. Cell line MANZ2-2. Scale $\operatorname{bar}=200 \mu \mathrm{m}$ 


\section{A}
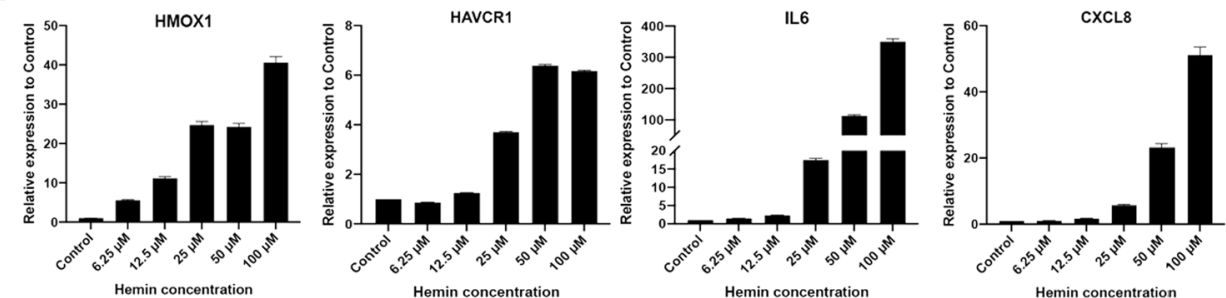

B
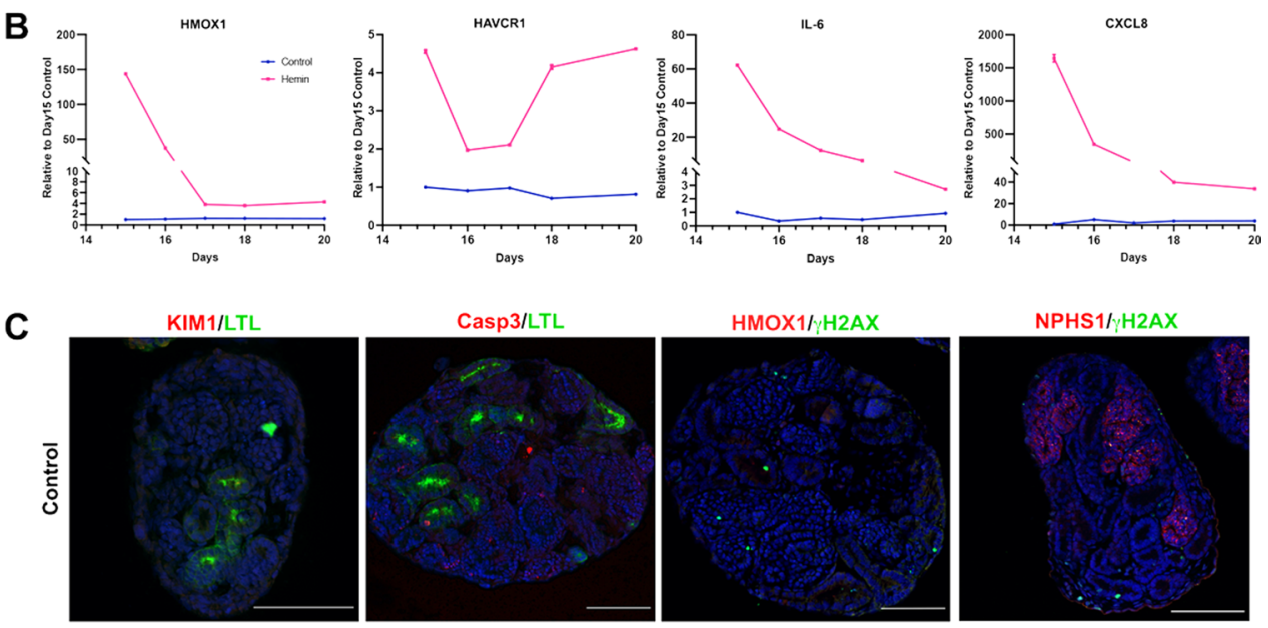

HMOX1/ $\mathrm{H} 2 \mathrm{AX}$

NPHS $1 / \gamma 2 \mathrm{HX}$

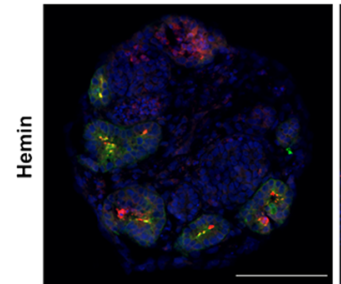

Day 16

D
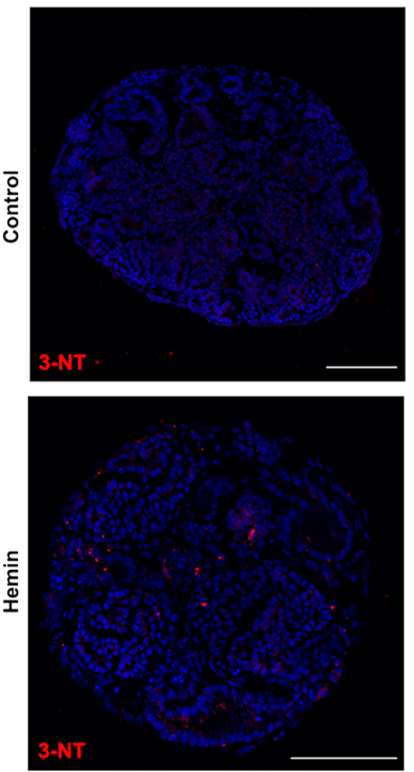

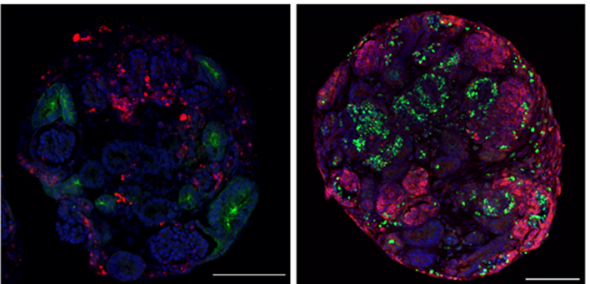

Day 18
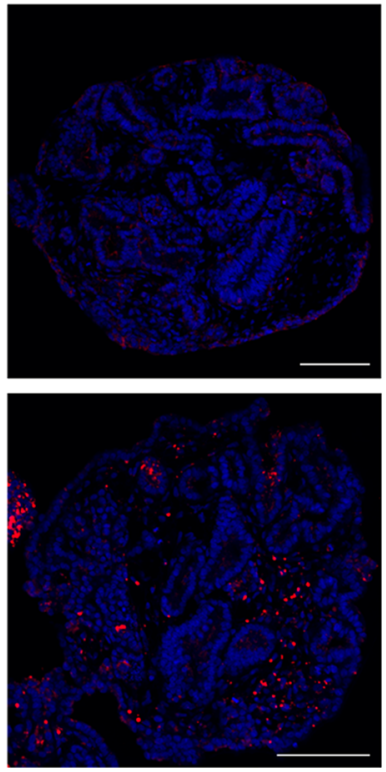

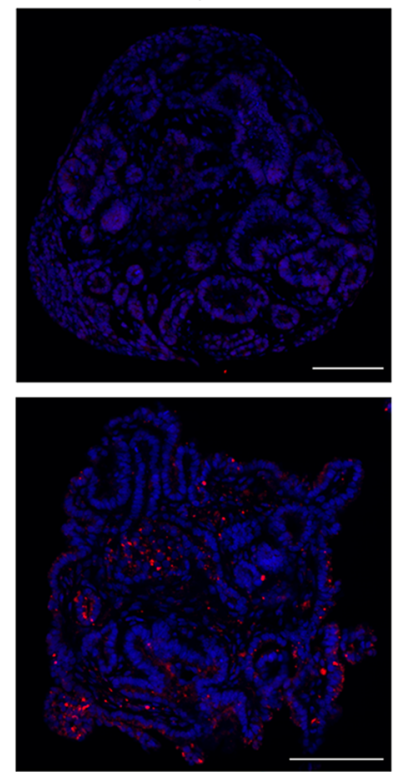

Fig. 1 (See legend on previous page.) 
controls (Fig. 1c; Additional file 1: Fig. S5). We found that staining for $\gamma \mathrm{H} 2 \mathrm{AX}$, a marker of double stranded DNA breaks that is induced by high levels of ROS, and caspase 3 (Casp3), labeling apoptotic cells, is also higher in the hemin-treated organoids compared to controls and was largely found in NPHS1-positive podocyte clusters (Fig. 1c). We next examined whether 3-nitrotyrosine, a marker of oxidative damage mediated by peroxynitrite, was increased in hemin treated organoids. We examined organoids treated at day 16,18 and 20 , and found very low levels of 3-nitrotyrosine in the control organoids. However, 3-nitrotyrosine was readily detected in hemin-treated organoids through day 20, where it appeared as punctate staining throughout the tissue, particularly in the stroma (Fig. 1d).

\section{Transcriptional analysis of hemin injury}

While the qPCR analysis of known injury markers demonstrated an injury response in hemin treated organoids, we wanted to assess transcriptional changes in response to hemin in a more comprehensive and unbiased fashion. To do this, we performed RNA-Seq on samples at days 15 and 16 (24 and $48 \mathrm{~h}$ post-hemin treatment, respectively; Fig. 2). Hallmark pathway analysis [52, 53] was performed to gain insight into the relevant biological processes being altered. The top pathways significantly upregulated at day 15 and 16 included those related to inflammation, apoptosis, and cellular stress (p53 pathway, hypoxia, UV response, unfolded protein response, reactive oxygen species; Additional file 1: Fig. S6). One major pathway that is induced in response to heme-induced stress is the protein degradation/ubiquitination pathway [54]. We found a number of proteasomal associated genes (HSPA1B, HSBP1, HSPA1A, HSP9OAB1) and UCHL5, $U B Q L N 1, U S P 14$ which are involved in the ubiquitination pathway [55], were significantly upregulated in the hemin-treated organoids on day 15 with lower, but still elevated, levels on day 16 (Fig. 2a). As expected, reactive oxygen species responsive genes (SOD2, SOD1, NQO1; Fig. 2b), and inflammatory markers IL6, CXCL8, NFKB1, NFKBIA, (Additional file 1: Fig. S7A) were also highly upregulated at day 15 . We also examined differences between day 15 and 16 hemin treated samples to determine if EMT and collagen induction was taking place. The major pathways that were overrepresented at day 16 were associated with cell cycle and EMT in hemin treated organoids (Fig. 2, Additional file 1: Fig. $\mathrm{S7B})$. In addition, a number of collagen and fibrosis related genes (COL16A1, FBN1, COL5A1, COL12A1, $A C T A 2, C O L 1 A 1, C O L 6 A 3)$ were upregulated at day 16
(Fig. 2d) suggesting initiation of early stages of fibrosis [56].

\section{Hemin injury leads to compromised function in the kidney organoids}

Since kidney organoids lack adequate vascularization and perfusion [25], transport throughout the nephron in the organoids is limited. However, we wanted to determine whether we could apply functional assays $[57,58]$ specifically adapted to test live kidney organoids and examine whether hemin-induced injury has an impact on organic anion and cation transport by the OAT and OCT transporters in the proximal tubule. We used a combined functional assay for proximal tubule transport of a fluorescent prototypical cation (ethidium bromide (EB)) and anion (6-carboxyfluorescein (6CF)) detected by fluorescence intensity and localization (e.g., luminal secretion or tubular uptake) (Fig. 3a). Kidney organoids were tested on day 16, after being treated for $48 \mathrm{~h}$ with hemin. We tested hemin concentrations of $12.5 \mu \mathrm{M}, 25 \mu \mathrm{M}$, and $50 \mu \mathrm{M}$ to confirm there was a concentration-dependent difference in function similar to what we observed with gene expression analysis (Figs. 1a, 3a). OAT function, as measured with $6 \mathrm{CF}$, decreased with increasing concentrations of hemin, with essentially no detectable uptake or secretion at $50 \mu \mathrm{M}$ hemin (Fig. 3a). OCT function, as measured with EB, showed decreased tubular fluorescence at $50 \mu \mathrm{M}$ hemin, and an increase in punctate fluorescence representative of EB intercalating into the double-stranded breaks in the DNA, marking apoptotic cells, throughout the kidney organoids at all doses (Fig. 3a) [59]. RNA-Seq analysis of specific basolateral (SLC22A2, SLC22A6, SLC22A8) and apical transporters (SLC47A1-A2, ABCC2) associated with organic anion and cation transport showed that they were all downregulated in the hemin-treated samples, except for $A B C C 2$, (coding for a multidrug resistant protein 2 - MRP2) which was higher in the hemin-treated samples. These results suggest that overall reduction in transport is associated with reduced gene expression of proximal tubule transporters, likely because of hemin-induced cellular injury. Additionally, the increase in $A B C C 2$ may suggest a greater cellular detoxification effort [60].

\section{Cytochrome-C-GFP biosensor to model injury in real-time}

For kidney organoids to be useful as a platform to screen drug toxicity or to treat renal injury, it would be ideal to assess early signs of apoptosis and injury via non-invasive, real-time monitoring. Towards this end we generated iPSC reporter lines that express CytochromeC-GFP (CytoC-GFP), a biosensor that is released from mitochondria upon cellular injury to assess apoptosis [15]. In healthy non-apoptotic cells, CytoC-GFP is associated 
A Color Key
and Histograr
Row Z-Score regulation of proteasomal ubiquitin-dependent protein
catabolic process
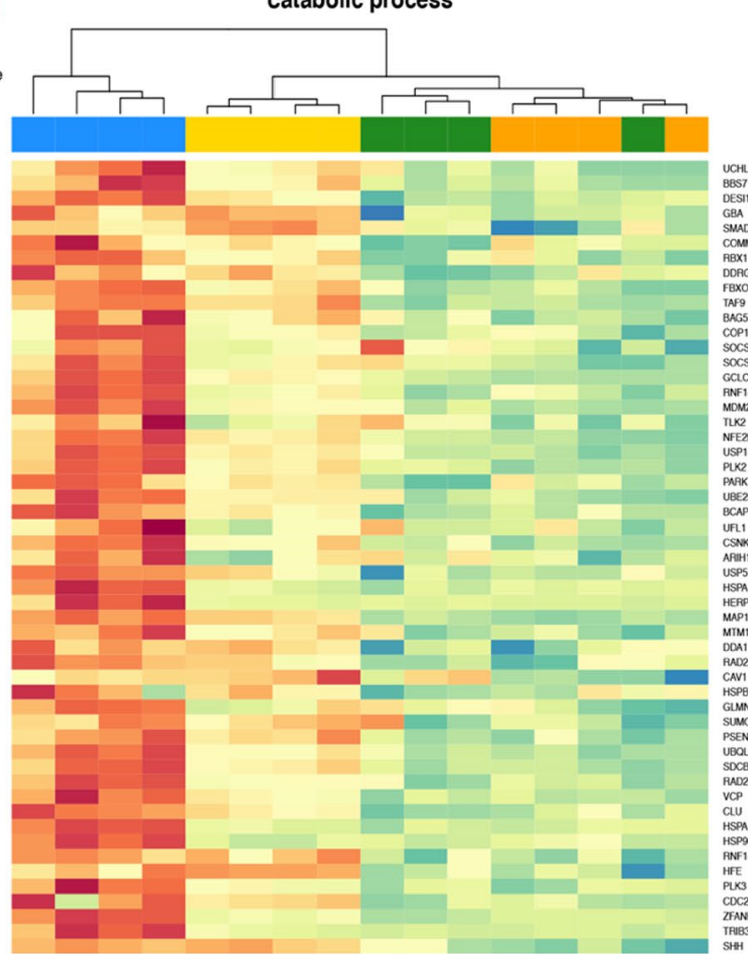

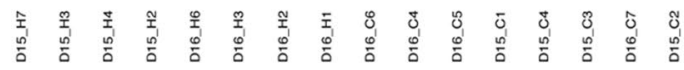

C

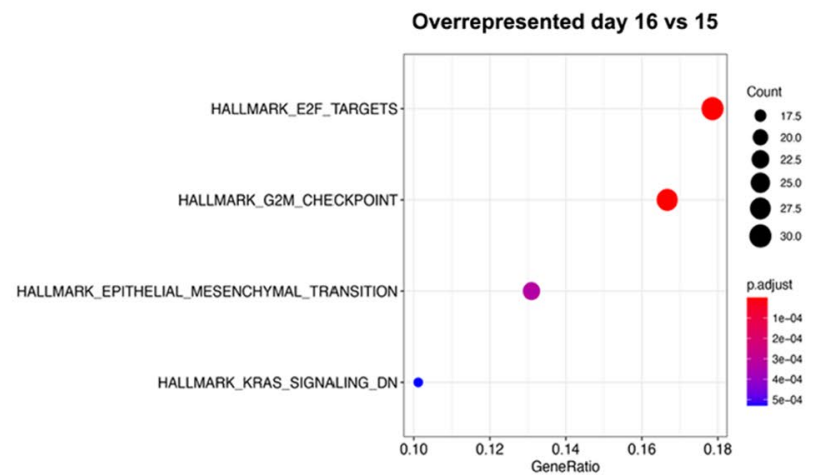

B

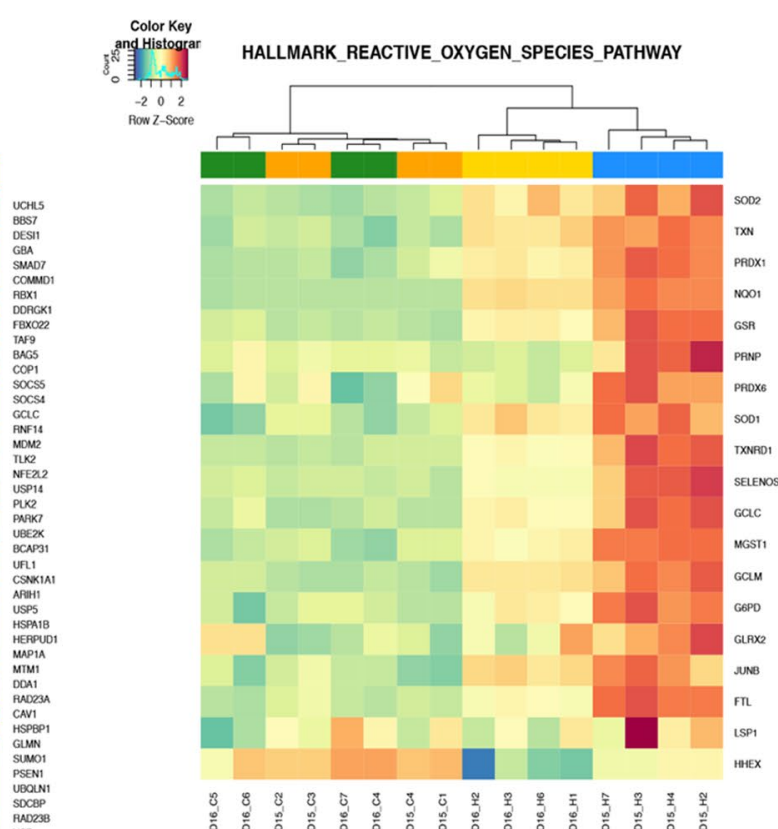

D

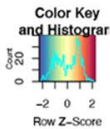

HALLMARK EPITHELIAL MESENCHYMAL TRANSITION
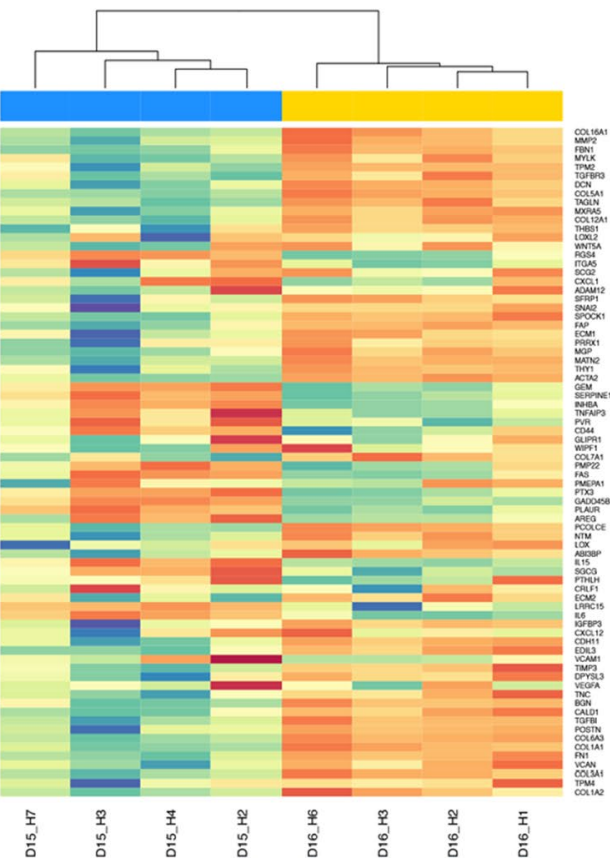

Fig. 2 Transcriptome analysis of hemin injured kidney organoids. Heatmaps showing z-score of differentially expressed genes between untreated control day 15 and 16 (_C) and hemin treated kidney organoids (_H). a GO Proteasomal ubiquitin dependent protein catabolic process. b Hallmark pathway analysis reactive oxygen species pathway. c Dot plot of overrepresented Hallmark pathways between days 16 and 15. d Heatmap of Hallmark epithelial-to-mesenchymal transition pathway showing differences between hemin-treated organoids day 15 and 16. Cell line MANZ2-2 


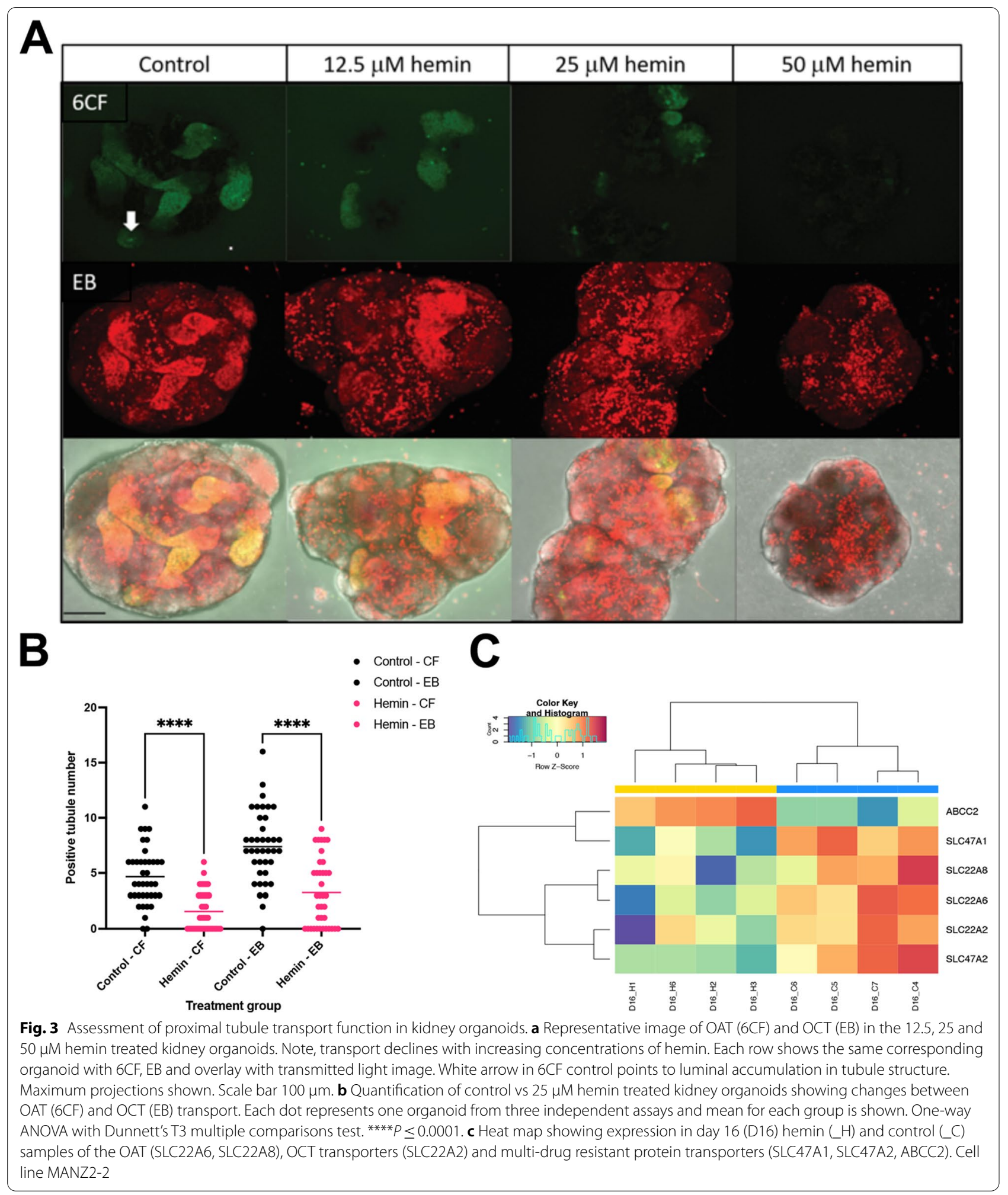

with mitochondria and shows a punctate or network-like fluorescence pattern. Upon cellular injury or increase in ROS, the fluorescence pattern rapidly shifts to being cytoplasmic. We confirmed that the CytoC-GFP signal in iPSCs was colocalized to the mitochondria by live coimmunofluorescence with MitoTracker Red CMXRos, 
a mitochondrial membrane potential dependent dye (Fig. 4b, Additional file 1: Fig. S8). Next, we generated kidney organoids from the CytoC-GFP iPSCs and treated them at day 14 with menadione, known to depolarize the mitochondrial membrane and induce apoptosis [61]. Menadione treated organoids exhibited diffuse cytoplasmic staining of CytoC-GFP in comparison to the untreated control (Fig. 4b, Additional file 1: Fig. S8). To confirm that inserting the CytoC-GFP transgene into the MANZ2-2 cell line did not increase the basal levels of ROS and apoptosis, we performed live staining with a superoxide indicator, dihydroethidium, which showed comparable levels of nuclear staining between the MANZ2-2 and CytoC-GFP cell lines (Additional file 1: Fig. S9A). Furthermore, levels of Casp3 were also comparable between the two cells lines (Fig. 1c, Additional file 1: Fig. S9B). To examine the effect of hemin treatment, CytoC-GFP kidney organoids were monitored over a 48-h window, post-hemin treatment. While control organoids showed CytoC-GFP signal in discrete elongated and interconnected tubular structures, consistent with mitochondria, hemin treatment induced foci of cells within the tubules and podocyte clusters with diffuse CytoC-GFP signal (Fig. 4c). These results suggest hemininduced ROS is driving apoptotic events, which are leading to mitochondrial dysfunction.

\section{Hemin-injured organoids show reduced fibrosis upon treatment with UPHD25}

ROS induced inflammation in the kidney leads to an upregulation of pro-inflammatory and pro-fibrotic cytokines, such as MCP1 and TGF- $\beta 1$, resulting in interstitial fibrosis [50]. We therefore tested if hemin treatment increased deposition of collagen in kidney organoids. Following a 48-h hemin treatment, hemin was washed out and kidney organoids were cultured to day 26. Based on previously published data [26], we know there is significant collagen deposition by day 26 , however with modifications of the protocol [27] there is less collagen seen at day 26 , thus allowing us to compare treated and untreated conditions at this timepoint. To determine the extent of the fibrotic response, we stained kidney organoid sections with collagen hybridizing peptide (CHP) which stains collagen undergoing remodeling or degradation seen in fibrotic diseases, and has been shown to be an accurate measure of organ fibrosis [62-64]. We found that there was a significant increase in CHP staining in hemin-treated kidney organoids compared to controls (Fig. 5a, b; Additional file 1: Fig. S10A, B). Furthermore, we performed staining with collagen 1a1 (COL1A1) and found the trend was comparable to CHP data (Fig. 5a, b; Additional file 1: Fig. S10A, B). To determine if the hemin injury model is responsive to therapeutic intervention, we examined whether the fibrotic response could be pharmacologically inhibited. In prior murine studies, we have shown that 4-(phenylthio)butanoic acid (PTBA) displays anti-fibrotic activity when delivered as a prodrug (UPHD25 or UPHD186) $[29,31]$. We therefore tested the effect of the methyl ester PTBA prodrug UPHD25 in hemin-injured kidney organoids. We tested doses of 1,3 and $9 \mu \mathrm{M}$ of UPHD25 in the hemin injury assay by delivering daily treatments from days 16-26 following hemin treatment from days 14-16. We tested two independent cell lines (MANZ2-2 (female origin) and MANZ4-37 (male origin)) and found that UPHD25 decreased CHP and COL1A1 staining at a concentration of $1 \mu \mathrm{M}$ in MANZ2-2 cell line, and decreased CHP staining with 3 and $9 \mu \mathrm{M}$ in MANZ4-37 (Fig. 5b, c. Additional file 1: Fig. S10B, C). These results suggest the fibrotic response in kidney organoids can be reduced with therapeutic intervention.

\section{Discussion}

Kidney organoids can be generated in large quantities, genetically manipulated, and have been shown to be a biologically relevant model for human disease [25]. Producing kidney organoids in bulk makes them well suited for testing therapeutic interventions [26]. While shortcomings with kidney organoid maturity are well-documented, [23] they are a good system to develop models for chronic organ diseases and fibrosis. Liver and lung organoids, which also display fetal like states comparable to early trimester development, have been used to study pulmonary and liver fibrosis [65].

Hemin is a potent inducer of ROS that mimics the hemolytic response seen in many diseases leading to renal injury [3]. Time course analysis of hemin-treated organoids revealed that the response to hemin injury was immediate and acute, with the inflammatory response and HMOX1 induction being rapidly downregulated within a couple of days of hemin removal, which is consistent for HMOX1 expression in injury settings [51].

\footnotetext{
(See figure on next page.)

Fig. 4 CytoC-GFP biosensor localizes to the mitochondria. a Schematic representation of the safe harbor AAVS1 donor vector with CytoC-GFP construct. $\mathbf{b}$ Live immunofluorescence showing CytoC-GFP expression is colocalized to MitoTracker Red CMXRos labelled mitochondria in the iPSCs and kidney organoids. Mitochondrial expression in the tubule of the kidney organoids, and diffuse cytoplasmic staining in the menadione treated tubule. Scale bar $=10 \mu \mathrm{m}$. c Representative live images of 3D projections showing mitochondrial localization in the control and $25 \mu \mathrm{M}$ hemin treated tubules of kidney organoids. Note the diffuse CytoC-GFP localization in the hemin treated tubule (white arrow). Data from isolated clone \#8 CytoC-GFP iPSC line. Scale bars $=20 \mu \mathrm{m}$
} 

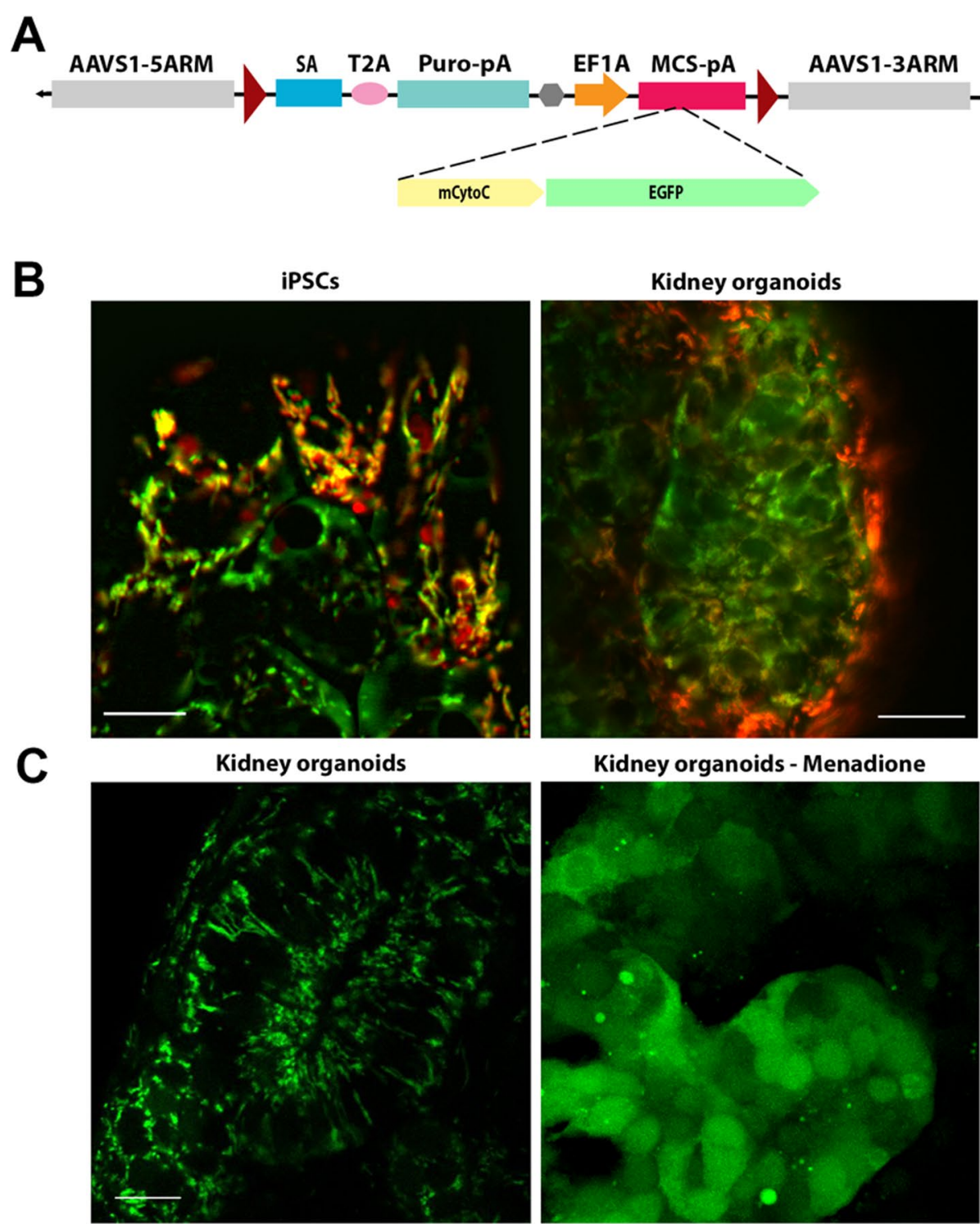

Kidney organoids - Menadione

D

Control

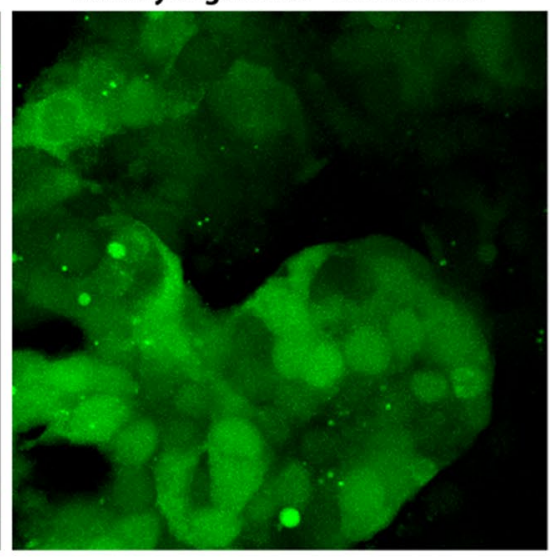

Hemin
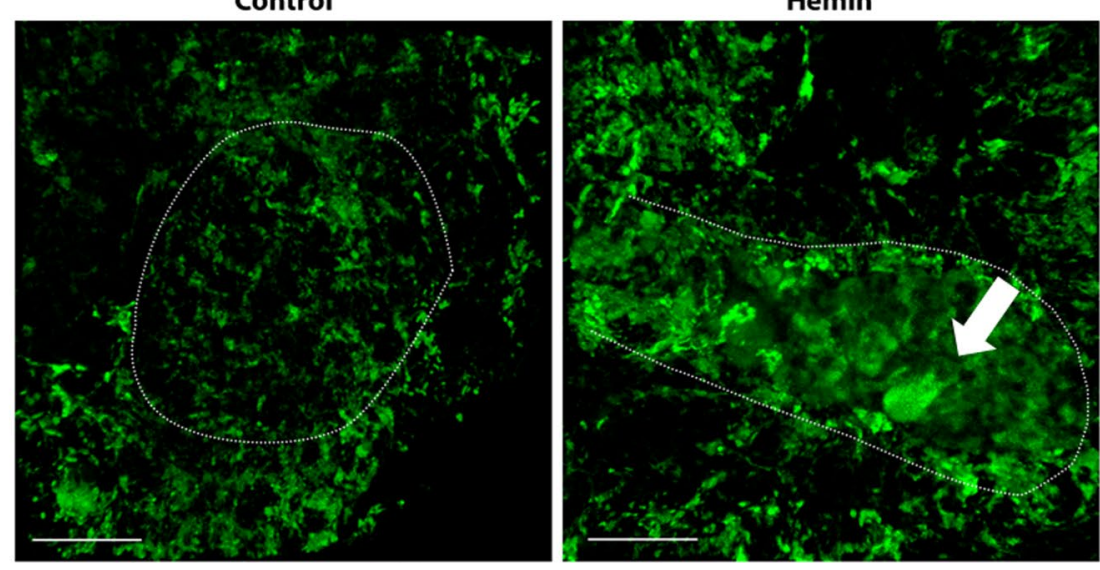

Fig. 4 (See legend on previous page.) 


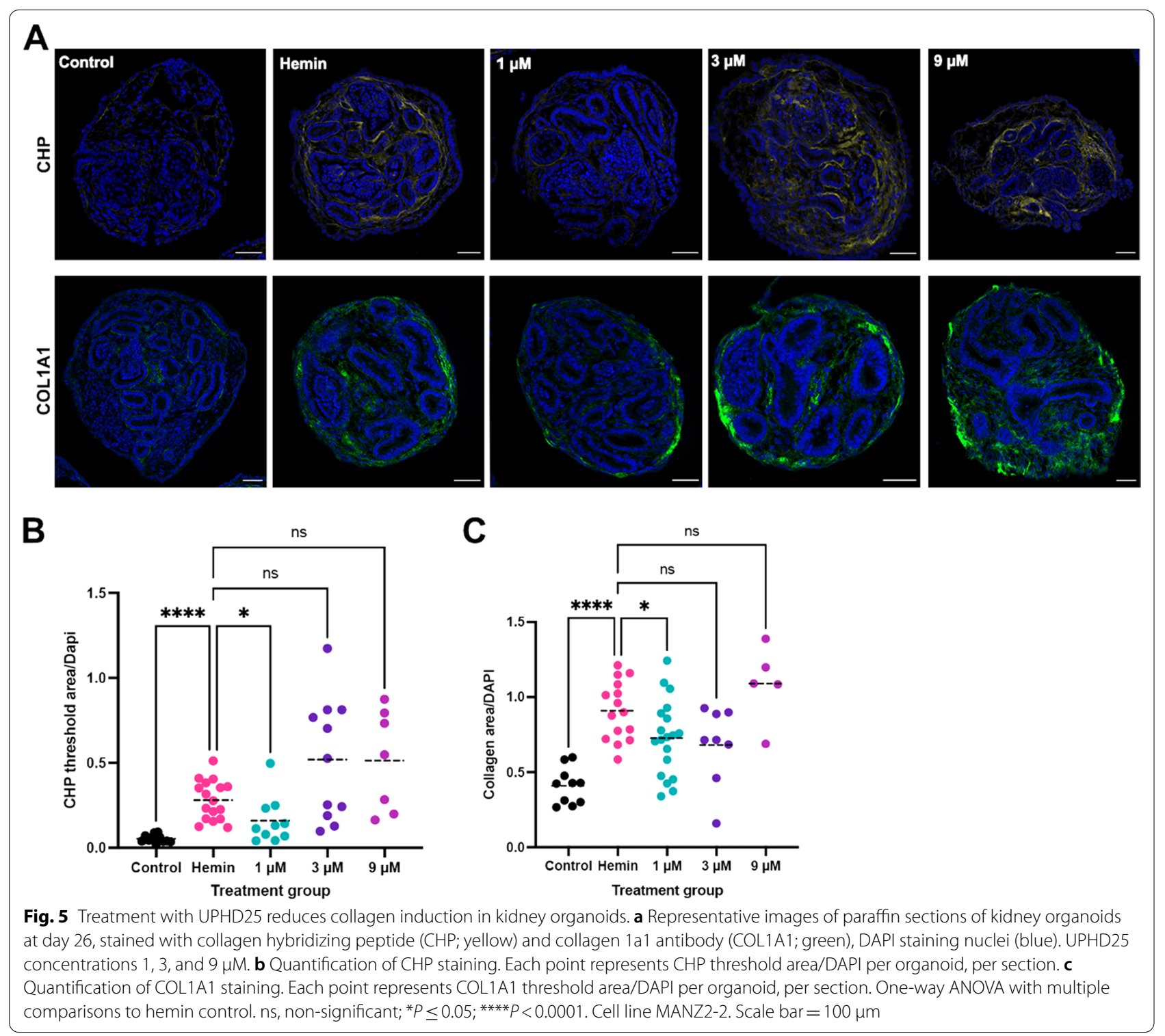

However, sustained HAVCR1 expression suggests that tubule cells undergo enduring injury in response to the brief hemin exposure. This is in accordance with other hemolytic models in mice which show a rapid decline of inflammatory markers but persistent expression of HAVCR1 [66]. Unilateral urothelial obstruction (UUO) and IRI injury models in mice also show that continued HAVCR1 expression is associated with higher levels of fibrosis and a progression to CKD [67, 68]. Elevated perdurance of HAVCR1 also translates to the clinic as not only do patients suffering from acute injury show elevated levels of urinary HAVCR1, but patients suffering from chronic injury show even higher levels of HAVCR1 [69].
High levels of ROS and nitrogen species are found during hemolytic events forming highly reactive peroxynitrite, and lead to nitrosylation of proteins [14]. We found that 3-nitrotyrosine (byproduct of nitration) was elevated in hemin treated organoids. In a condition unrelated to hemin induced injury, patients with septic shock and CKD show high levels of nitrotyrosine as well as nitrite/nitrate (indicating NO production) levels in plasma, suggesting the nitration of tyrosine residues is directly related to disease causing events [70]. In addition, rats loaded with iron exhibited nitrotyrosine labeled tubules and interstitial cells. They had elevated levels of NO synthase enzymes (responsible for NO production), indicating increased levels of 
reactive nitrogen species due to high levels of iron [71]. This work supports the notion that high peroxynitrite levels and nitric oxide levels can play a critical role in hemolytic events and the subsequent injury. Another major cellular response to high oxidative stress is an increase in ubiquitination and proteasomal degradation of proteins [72]. Our RNA-Seq analysis revealed the top differentially expressed genes were part of the ubiquitin-proteasome degradation network and one of the overrepresented pathways was the unfolded protein response, suggesting the hemin treated organoids were trying to eliminate unfolded or damaged proteins in order to ameliorate cellular injury [73].

Inhibition of the proteasome system leads to an increase in ubiquitination of mitochondrial proteins and recruitment of heat shock, and autophagy related components [74]. Therefore, mitochondria need to be continuously monitored during ROS events for potential dysfunction. We developed the CytoC-GFP iPSC line as a real-time biosensor, which can be utilized to monitor mitochondrial health, morphology, and cellular response to toxins [15]. Recent studies show that CytoC may have other functions beyond apoptosis induction, which could further aid in understanding injury mechanisms. CytoC has been shown to translocate to the nucleus before activating the apoptotic cascade, where the protein plays a role in the DNA damage repair response $[75,76]$. This suggests that not all cells that release CytoC from the mitochondria undergo apoptosis. Dissecting this mechanism further, may shed light on the repair pathways and potential future treatments. Integration with new imaging technologies and other biosensors may further our understanding of cellular injury particularly in PT cells which are rich in mitochondria [77].

Finally, we show that our model of hemin mediated injury can be adapted to therapeutic compound testing by showing a reduction in the fibrotic response. Previous studies have shown that PTBA, delivered as a prodrug, can ameliorate injury and reduce fibrosis in multiple models of renal injury [29-31]. As with most potential renal therapeutics, PTBA has never been validated against a human proteome, especially in an injury setting. In addition, when performing therapeutic injury studies, several shortcomings need to be considered. Kidney organoids are immature and are more closely related to embryonic kidney epithelium and interstitium [78]. In addition, longer-term injury studies are currently not feasible, as organoids lack a blood supply, filtration, or tubular flow, and are lacking an immune system, which may not allow modeling of some of the molecular and cellular responses seen in injured adult kidneys [79]. However, organoids still have the potential to be useful for studying the efficacy and safety of potential therapeutics, as they provide the advantage of multiple cell types and structures over standard 2D culture systems [80].

\section{Conclusions}

This work demonstrates we can model aspects of renal injury such as fibrosis and pro-inflammatory cytokine expression, and therapeutically intervene to reduce the injury and possibly stimulate repair. For the scientific community to take full advantage of kidney organoid systems, we need to further our understanding of the injury processes that take place in organoids and how they compare to in vivo kidney injury. This includes understanding the repair process that occurs after the initial insult and the longer-term outcomes. The hemin injury model displays many of the same hallmarks as in vivo hemolytic injury, and may allow validation of the efficacy of targeted renal therapeutics in a human model of renal injury. However, as with any preclinical model there are limitations, and while this model maybe a good model of cellular injury, due to the limitations of the kidney organoids it cannot model other hemin mediated injury events such as tubular obstruction or vasoconstriction.

\begin{abstract}
Abbreviations
6CF: 6-Carboxyfluorescein; ACO1: Aconitase 1; ALAD: Aminolevulinate dehydratase; AKI: Acute kidney injury; BLVR: Biliverdin reductase; CHP: Collagen hybridizing peptide; CKD: Chronic kidney disease; COL_A_: Collagen Type_ Alpha_; CXCL8: C-X-C Motif Chemokine Ligand 8; CytoC-GFP: Cytochrome C-GFP; DE: Differential expression; EB: Ethidium bromide; FBN1: Fibrillin 1; FA: Formic acid; HAVCR1: Hepatitis A virus cellular receptor 1; HDAC: Histone deacetylase; HEBP: Heme binding protein; HMBS: Hydroxymethylbilane synthase; HMOX1: Heme oxygenase 1; HRG: Histidine rich glycoprotein; IL6: Interleukin 6; iPSCs: Induced pluripotent stem cells; IRI: Ischemia reperfusion injury; LRP: LDL receptor related protein; LTL: Lotus tetragonolobus lectin; MCP1: Monocyte chemoattractant protein-1; MRP2: Multidrug resistant protein 2; NO: Nitric oxide; NQO1: NAD(P)H quinone dehydrogenase 1; OAT: Organic anion transporter; OCT: Organic cation transporter; PTBA: 4-(Phenylthio) butanoic; ROS: Reactive oxygen species; SLC_: Solute Carrier Family_Member; SOD: Superoxide dismutase 1;TFRC: Transferrin receptor; TGF- $\beta 1$ : Transforming growth factor beta 1; UCHL5: Ubiquitin C-terminal hydrolase L5; UBQLN1: Ubiquilin 1; USP14: Ubiquitin specific peptidase 14; ULA: Ultra-low attachment; UROD: Uroporphyrinogen decarboxylase; UUO: Unilateral urothelial obstruction.
\end{abstract}

\section{Supplementary Information}

The online version contains supplementary material available at https://doi. org/10.1186/s13287-022-02752-z.

Additional file 1. Supplementary Figures.

\section{Acknowledgements}

We would like to thank Michele Mulkeen for histology sectioning, and Rania Elbakri and William A. MacDonald from Health Sciences Sequencing Core, UPMC Children's Hospital of Pittsburgh for assistance with RNA-Seq experiments.

\section{Authors' contributions}

AP, TV, EBE, AEC, DRE, LAV, MLM, JAK, TRK, CJB, AJD, NAH—designed research studies; AP, EBE, AEC, EP, MDM, RS, CLH, CJB-conducted experiments; AP, $E B E, A E C, R S, C L H, C J B$ - acquired data; AP, TV, EBE, AEC, MLM, TRK, CJB, AJD, 
$\mathrm{NAH}$ - analyzed data; and AP, AEC, CJB, NAH—wrote the manuscript. All authors read and approved the final manuscript.

\section{Funding}

This research was funded by the National Institutes of Health [2R01DK069403, 2UC2DK126122, to N.A.H., 1P30DK079307 to T,R.K., and T32DK061296 to E.B.E.]; the U.S. Department of Defense [W81XWH-17-1-0610 to N.A.H.]; the American Society of Nephrology Ben J. Lipps Award to A.P.

\section{Availability of data and materials}

All relevant data have been uploaded as part of supplementary files and available on National Center for Biotechnology Information's GEO database.

\section{Declarations}

\section{Ethics approval and consent to participate}

All work was performed in compliance with institutional guidelines (IBC201600244).

\section{Consent for publication}

Not applicable.

\section{Competing Interests}

The authors declare that they have no competing interests.

\section{Author details}

1 Department of Developmental Biology, University of Pittsburgh, School of Medicine, 3501 5th Ave., 5061 BST3, Pittsburgh, PA 15213, USA. ²Department of Molecular Medicine and Pathology, School of Medical Sciences, University of Auckland, Auckland, New Zealand. ${ }^{3}$ Center for Critical Care Nephrology, University of Pittsburgh, School of Medicine, 3501 5th Ave., 5061 BST3, Pittsburgh, PA 15213, USA. " Department of Psychiatry, University of Pittsburgh, School of Medicine, 3501 5th Ave., 5061 BST3, Pittsburgh, PA 15213, USA.

${ }^{5}$ Department of Computational and Systems Biology, University of Pittsburgh, School of Medicine, 3501 5th Ave., 5061 BST3, Pittsburgh, PA 15213, USA

${ }^{6}$ Renal-Electrolyte Division, Department of Medicine, University of Pittsburgh, School of Medicine, 3501 5th Ave., 5061 BST3, Pittsburgh, PA 15213, USA.

\section{Received: 18 August 2021 Accepted: 8 February 2022}

Published online: 21 February 2022

\section{References}

1. Haase M, Bellomo R, Haase-Fielitz A. Novel biomarkers, oxidative stress, and the role of labile iron toxicity in cardiopulmonary bypass-associated acute kidney injury. J Am Coll Cardiol. 2010;55(19):2024-33.

2. Reiter CD, Wang X, Tanus-Santos JE, Hogg N, Cannon RO 3rd, Schechter AN, Gladwin MT. Cell-free hemoglobin limits nitric oxide bioavailability in sickle-cell disease. Nat Med. 2002;8(12):1383-9.

3. Rother RP, Bell L, Hillmen P, Gladwin MT. The clinical sequelae of intravascular hemolysis and extracellular plasma hemoglobin: a novel mechanism of human disease. JAMA. 2005:293(13):1653-62.

4. Wang L, Vijayan V, Jang MS, Thorenz A, Greite R, Rong S, Chen R, Shushakova N, Tudorache I, Derlin K, et al. Labile heme aggravates renal inflammation and complement activation after ischemia reperfusion injury. Front Immunol. 2019;10:2975

5. Vallelian F, Schaer CA, Deuel JW, Ingoglia G, Humar R, Buehler PW, Schaer DJ. Revisiting the putative role of heme as a trigger of inflammation. Pharmacol Res Perspect. 2018;6(2):e00392.

6. Malle E, Woenckhaus C, Waeg G, Esterbauer H, Grone EF, Grone HJ. Immunological evidence for hypochlorite-modified proteins in human kidney. Am J Pathol. 1997;150(2):603-15.

7. Antolini F, Valente F, Ricciardi D, Baroni M, Fagugli RM. Principal component analysis of some oxidative stress parameters and their relationships in hemodialytic and transplanted patients. Clin Chim Acta. 2005;358(1-2):87-94.

8. Jeney V, Balla J, Yachie A, Varga Z, Vercellotti GM, Eaton JW, Balla G. Pro-oxidant and cytotoxic effects of circulating heme. Blood. 2002:100(3):879-87.
9. Raatikainen-Ahokas A, Hytönen M, Tenhunen A, Sainio K, Sariola H. BMP-4 affects the differentiation of metanephric mesenchyme and reveals an early anterior-posterior axis of the embryonic kidney. Dev Dyn. 2000;217(2):146-58

10. Gutteridge JMC. Iron promoters of the Fenton reaction and lipid peroxidation can be released from haemoglobin by peroxides. FEBS Lett. 1986;201(2):291-5.

11. Ushio-Fukai M, Ash D, Nagarkoti S. Belin de Chantemele EJ, Fulton DJR, Fukai T: Interplay between reactive oxygen/reactive nitrogen species and metabolism in vascular biology and disease. Antioxid Redox Signal. 2021;34(16):1319-54.

12. Nath KA, Grande JP, Croatt AJ, Likely S, Hebbel RP, Enright H. Intracellular targets in heme protein-induced renal injury. Kidney Int. 1998:53(1):100-11.

13. Balabanli B, Kamisaki Y, Martin E, Murad F. Requirements for heme and thiols for the nonenzymatic modification of nitrotyrosine. Proc Natl Acad Sci U S A. 1999;96(23):13136-41.

14. MacMillan-Crow LA, Crow JP, Kerby JD, Beckman JS, Thompson JA. Nitration and inactivation of manganese superoxide dismutase in chronic rejection of human renal allografts. Proc Natl Acad Sci U S A. 1996;93(21):11853-8.

15. Goldstein JC, Waterhouse NJ, Juin P, Evan Gl, Green DR. The coordinate release of cytochrome $\mathrm{C}$ during apoptosis is rapid, complete and kinetically invariant. Nat Cell Biol. 2000;2(3):156-62.

16. Min $L$, Jian-xing $X$. Detoxifying function of cytochrome $C$ against oxygen toxicity. Mitochondrion. 2007;7(1-2):13-6.

17. Cai J, Yang J, Jones DP. Mitochondrial control of apoptosis: the role of cytochrome C. Biochim Biophys Acta. 1998;1366(1-2):139-49.

18. de Caestecker M, Harris R. Translating knowledge into therapy for acute kidney injury. Semin Nephrol. 2018;38(1):88-97.

19. Digby JLM, Vanichapol T, Przepiorski A, Davidson AJ, Sander V. Evaluation of cisplatin-induced injury in human kidney organoids. Am J Physiol Renal Physiol. 2020;318(4):F971-8.

20. Higgins JW, Chambon A, Bishard K, Hartung A, Arndt D, Brugnano J, Er PX Lawlor KT, Vanslambrouck JM, Wilson S et al: Bioprinted pluripotent stem cell-derived kidney organoids provide opportunities for high content screening. bioRxiv 2018:505396.

21. Uchimura K, Wu H, Yoshimura Y, Humphreys BD. Human pluripotent stem cell-derived kidney organoids with improved collecting duct maturation and injury modeling. Cell Rep. 2020;33(11):108514.

22. Czerniecki SM, Cruz NM, Harder JL, Menon R, Annis J, Otto EA, Gulieva RE, Islas LV, Kim YK, Tran LM, et al. High-throughput screening enhances kidney organoid differentiation from human pluripotent stem cells and enables automated multidimensional phenotyping. Cell Stem Cell. 2018;22(6):929-40.

23. Takasato M, Er PX, Chiu HS, Maier B, Baillie GJ, Ferguson C, Parton RG, Wolvetang EJ, Roost MS, Lopes SM, et al. Kidney organoids from human iPS cells contain multiple lineages and model human nephrogenesis. Nature. 2016;536(7615):238

24. Morizane R, Lam AQ, Freedman BS, Kishi S, Valerius MT, Bonventre JV. Nephron organoids derived from human pluripotent stem cells model kidney development and injury. Nat Biotechnol. 2015;33(11):1193-200.

25. Przepiorski A, Crunk AE, Espiritu EB, Hukriede NA, Davidson AJ. The utility of human kidney organoids in modeling kidney disease. Semin Nephrol. 2020;40(2):188-98.

26. Przepiorski A, Sander V, Tran T, Hollywood JA, Sorrenson B, Shih JH, Wolvetang EJ, McMahon AP, Holm TM, Davidson AJ. A simple bioreactor-based method to generate kidney organoids from pluripotent stem cells. Stem Cell Rep. 2018;11(2):470-84

27. Przepiorski A, Crunk AE, Holm TM, Sander V, Davidson AJ, Hukriede NA. A simplified method for generating kidney organoids from human pluripotent stem cells. J Vis Exp. 2021. https://doi.org/10.3791/62452.

28. Brilli Skvarca L, Han HI, Espiritu EB, Missinato MA, Rochon ER, McDaniels MD, Bais AS, Roman BL, Waxman JS, Watkins SC, et al. Enhancing regeneration after acute kidney injury by promoting cellular dedifferentiation in zebrafish. Dis Model Mech. 2019. https://doi.org/10.1242/dmm.037390.

29. Cianciolo Cosentino C, Skrypnyk NI, Brilli LL, Chiba T, Novitskaya T, Woods C, West J, Korotchenko VN, McDermott L, Day BW, et al. Histone deacetylase inhibitor enhances recovery after AKI. J Am Soc Nephrol. 2013;24(6):943-53. 
30. Novitskaya T, McDermott L, Zhang KX, Chiba T, Paueksakon P, Hukriede NA, de Caestecker MP. A PTBA small molecule enhances recovery and reduces postinjury fibrosis after aristolochic acid-induced kidney injury. Am J Physiol Renal Physiol. 2014;306(5):F496-504.

31. Skrypnyk NI, Sanker S, Skvarca LB, Novitskaya T, Woods C, Chiba T, Patel K, Goldberg ND, McDermott L, Vinson PN, et al. Delayed treatment with PTBA analogs reduces postinjury renal fibrosis after kidney injury. Am J Physiol Renal Physiol. 2016;310(8):F705-16.

32. Wen X, Li S, Frank A, Chen X, Emlet D, Hukriede NA, Kellum JA. Timedependent effects of histone deacetylase inhibition in sepsis-associated acute kidney injury. Intensive Care Med Exp. 2020;8(1):9.

33. Oh JK, Przepiorski A, Chang H-H, Dodd RC, Sander V, Sorrenson B, Shih J-H, Hollywood JA, de Zoysa JR, Shepherd PR, et al. Derivation of induced pluripotent stem cell lines from New Zealand donors. J Royal Soc New Zealand. 2022;52(1):54-67. https://doi.org/10.1080/03036758.2020.18308 08.

34. Manissorn J, Fong-Ngern K, Peerapen P, Thongboonkerd V. Systematic evaluation for effects of urine $\mathrm{pH}$ on calcium oxalate crystallization, crystal-cell adhesion and internalization into renal tubular cells. Sci Rep. 2017:7(1):1798.

35. Livak KJ, Schmittgen TD. Analysis of relative gene expression data using real-time quantitative PCR and the 2(-Delta Delta C(T)) method. Methods. 2001;25(4):402-8.

36. Dobin A, Davis CA, Schlesinger F, Drenkow J, Zaleski C, Jha S, Batut P, Chaisson M, Gingeras TR. STAR: ultrafast universal RNA-seq aligner. Bioinformatics. 2013;29(1):15-21.

37. Liao Y, Smyth GK, Shi W. featureCounts: an efficient general purpose program for assigning sequence reads to genomic features. Bioinformatics. 2014;30(7):923-30.

38. Robinson MD, McCarthy DJ, Smyth GK. edgeR: a bioconductor package for differential expression analysis of digital gene expression data. Bioinformatics. 2010;26(1):139-40.

39. Ritchie ME, Phipson B, Wu D, Hu Y, Law CW, Shi W, Smyth GK. limma powers differential expression analyses for RNA-sequencing and microarray studies. Nucleic Acids Res. 2015;43(7):e47.

40. Ashburner M, Ball CA, Blake JA, Botstein D, Butler H, Cherry JM, Davis AP, Dolinski K, Dwight SS, Eppig JT, et al. Gene ontology: tool for the unification of biology. The Gene Ontology Consortium. Nat Genet. 2000;25(1):25-9.

41. Liberzon A, Birger C, Thorvaldsdottir H, Ghandi M, Mesirov JP, Tamayo P. The Molecular Signatures Database (MSigDB) hallmark gene set collection. Cell Syst. 2015;1(6):417-25.

42. Gene Ontology C. The Gene Ontology resource: enriching a GOld mine. Nucleic Acids Res. 2021:49(D1):D325-34.

43. Yu G, Wang LG, Han Y, He QY. clusterProfiler: an R package for comparing biological themes among gene clusters. OMICS. 2012;16(5):284-7.

44. Zecha J, Satpathy S, Kanashova T, Avanessian SC, Kane MH, Clauser KR, Mertins P, Carr SA, Kuster B. TMT labeling for the masses: a robust and cost-efficient, in-solution labeling approach. Mol Cell Proteomics. 2019;18(7):1468-78.

45. Jiang B, Liu G, Zheng J, Chen M, Maimaitiming Z, Chen M, Liu S, Jiang R, Fuqua BK, Dunaief $J L$, et al. Hephaestin and ceruloplasmin facilitate iron metabolism in the mouse kidney. Sci Rep. 2016;6:39470.

46. Bednarz A, Lipinski P, Starzynski RR, Tomczyk M, Nowak W, Mucha O, Ogorek M, Pierzchala O, Jonczy A, Staron R, et al. Role of the kidneys in the redistribution of heme-derived iron during neonatal hemolysis in mice. Sci Rep. 2019;9(1):11102.

47. van Raaij S, van Swelm R, Bouman K, Cliteur M, van den Heuvel MC, Pertijs $J$, Patel D, Bass P, van Goor H, Unwin R, et al. Tubular iron deposition and iron handling proteins in human healthy kidney and chronic kidney disease. Sci Rep. 2018;8(1):9353.

48. Gonzalez-Michaca L, Farrugia G, Croatt AJ, Alam J, Nath KA. Heme: a determinant of life and death in renal tubular epithelial cells. Am J Physiol Renal Physiol. 2004;286(2):F370-377.

49. Goldstein L, Teng ZP, Zeserson E, Patel M, Regan RF. Hemin induces an iron-dependent, oxidative injury to human neuron-like cells. J Neurosci Res. 2003;73(1):113-21.

50. Van Avondt K, Nur E, Zeerleder S. Mechanisms of haemolysis-induced kidney injury. Nat Rev Nephrol. 2019;15(11):671-92.
51. Young R, Wolf CR, Brown K, Hayes JD, Whitelaw CB. Spatial monitoring of toxicity in HMOX-LacZ transgenic mice. Transgenic Res. 2010;19(5):897-902.

52. Subramanian A, Tamayo P, Mootha VK, Mukherjee S, Ebert BL, Gillette MA, Paulovich A, Pomeroy SL, Golub TR, Lander ES, et al. Gene set enrichment analysis: a knowledge-based approach for interpreting genome-wide expression profiles. Proc Natl Acad Sci U S A. 2005;102(43):15545-50.

53. Mootha VK, Lindgren CM, Eriksson KF, Subramanian A, Sihag S, Lehar J, Puigserver P, Carlsson E, Ridderstrale M, Laurila E, et al. PGC-1alpharesponsive genes involved in oxidative phosphorylation are coordinately downregulated in human diabetes. Nat Genet. 2003;34(3):267-73.

54. Vasconcellos LR, Dutra FF, Siqueira MS, Paula-Neto HA, Dahan J, Kiarely E, Carneiro LA, Bozza MT, Travassos LH. Protein aggregation as a cellular response to oxidative stress induced by heme and iron. Proc Natl Acad Sci U S A. 2016;113(47):E7474-82.

55. Whiteley AM, Prado MA, Peng I, Abbas AR, Haley B, Paulo JA, Reichelt M, Katakam A, Sagolla M, Modrusan Z, et al. Ubiquilin1 promotes antigenreceptor mediated proliferation by eliminating mislocalized mitochondrial proteins. Elife. 2017. https://doi.org/10.7554/eLife.26435.

56. Canaud G, Brooks CR, Kishi S, Taguchi K, Nishimura K, Magassa S, Scott A, Hsiao LL, Ichimura T, Terzi F, et al. Cyclin G1 and TASCC regulate kidney epithelial cell G2-M arrest and fibrotic maladaptive repair. Sci TransI Med. 2019. https://doi.org/10.1126/scitranslmed.aav4754.

57. Lee WK, Reichold M, Edemir B, Ciarimboli G, Warth R, Koepsell H, Thevenod F. Organic cation transporters OCT1, 2, and 3 mediate highaffinity transport of the mutagenic vital dye ethidium in the kidney proximal tubule. Am J Physiol Renal Physiol. 2009;296(6):F1504-1513.

58. Lawrence ML, Chang CH, Davies JA. Transport of organic anions and cations in murine embryonic kidney development and in seriallyreaggregated engineered kidneys. Sci Rep. 2015;5:9092.

59. Ribble D, Goldstein NB, Norris DA, Shellman YG. A simple technique for quantifying apoptosis in 96-well plates. BMC Biotechnol. 2005;5:12.

60. Faucher Q, Alarcan H, Marquet P, Barin-Le Guellec C. Effects of ischemiareperfusion on tubular cell membrane transporters and consequences in kidney transplantation. J Clin Med. 2020;9(8):2610.

61. Gerasimenko JV, Gerasimenko OV, Palejwala A, Tepikin AV, Petersen $\mathrm{OH}$, Watson AJ. Menadione-induced apoptosis: roles of cytosolic $\mathrm{Ca}(2+)$ elevations and the mitochondrial permeability transition pore. J Cell Sci. 2002;115(Pt 3):485-97.

62. Li Y, Ho D, Meng H, Chan TR, An B, Yu H, Brodsky B, Jun AS, Michael YuS. Direct detection of collagenous proteins by fluorescently labeled collagen mimetic peptides. Bioconjug Chem. 2013;24(1):9-16.

63. Hwang J, Huang Y, Burwell TJ, Peterson NC, Connor J, Weiss SJ, Yu SM, Li Y. In situ imaging of tissue remodeling with collagen hybridizing peptides. ACS Nano. 2017;11(10):9825-35.

64. Jaramillo C, Guthery SL, Lowichik A, Stoddard G, Kim T, Li Y, Jensen MK. Quantitative liver fibrosis using collagen hybridizing peptide to predict native liver survival in biliary atresia: a pilot study. J Pediatr Gastroenterol Nutr. 2020;70(1):87-92.

65. Lee J, Kim JH, Hong SH, Yang SR. Organoid model in idiopathic pulmonary fibrosis. Int J Stem Cells. 2021;14(1):1-8.

66. Merle NS, Grunenwald A, Figueres ML, Chauvet S, Daugan M, Knockaert S, Robe-Rybkine T, Noe R, May O, Frimat M, et al. Characterization of renal injury and inflammation in an experimental model of intravascular hemolysis. Front Immunol. 2018;9:179.

67. Humphreys BD, Xu F, Sabbisetti V, Grgic I, Movahedi Naini S, Wang N, Chen G, Xiao S, Patel D, Henderson JM, et al. Chronic epithelial kidney injury molecule-1 expression causes murine kidney fibrosis. J Clin Invest. 2013;123(9):4023-35.

68. Ko GJ, Grigoryev DN, Linfert D, Jang HR, Watkins T, Cheadle C, Racusen L, Rabb H. Transcriptional analysis of kidneys during repair from AKI reveals possible roles for NGAL and KIM-1 as biomarkers of AKI-to-CKD transition. Am J Physiol Renal Physiol. 2010;298(6):F1472-1483.

69. Tian L, Shao X, Xie Y, Wang Q, Che X, Zhang M, Xu W, Xu Y, Mou S, Ni Z. Kidney injury molecule-1 is elevated in nephropathy and mediates macrophage activation via the mapk signalling pathway. Cell Physiol Biochem. 2017;41(2):769-83.

70. Fukuyama N, Takebayashi Y, Hida M, Ishida H, Ichimori K, Nakazawa $\mathrm{H}$. Clinical evidence of peroxynitrite formation in chronic renal failure patients with septic shock. Free Radic Biol Med. 1997;22(5):771-4. 
71. Zhou XJ, Laszik Z, Wang XQ, Silva FG, Vaziri ND. Association of renal injury with increased oxygen free radical activity and altered nitric oxide metabolism in chronic experimental hemosiderosis. Lab Invest. 2000;80(12):1905-14.

72. Shang F, Taylor A. Ubiquitin-proteasome pathway and cellular responses to oxidative stress. Free Radic Biol Med. 2011;51(1):5-16.

73. Vallelian F, Deuel JW, Opitz L, Schaer CA, Puglia M, Lonn M, Engelsberger W, Schauer S, Karnaukhova E, Spahn DR, et al. Proteasome inhibition and oxidative reactions disrupt cellular homeostasis during heme stress. Cell Death Differ. 2015;22(4):597-611.

74. Sulkshane P, Duek I, Ram J, Thakur A, Reis N, Ziv T, Glickman MH. Inhibition of proteasome reveals basal mitochondrial ubiquitination. J Proteomics. 2020;229:103949

75. Diaz-Moreno I, Velazquez-Cruz A, Curran-French S, Diaz-Quintana A, De la Rosa MA. Nuclear cytochrome C - a mitochondrial visitor regulating damaged chromatin dynamics. FEBS Lett. 2018;592(2):172-8.

76. Rivero-Rodriguez F, Diaz-Quintana A, Velazquez-Cruz A, Gonzalez-Arzola K, Gavilan MP, Velazquez-Campoy A, Rios RM, De la Rosa MA, Diaz-Moreno I. Inhibition of the PP2A activity by the histone chaperone ANP32B is long-range allosterically regulated by respiratory cytochrome C. Redox Biol. 2021;43:101967.

77. Okkelman IA, Papkovsky DB, Dmitriev RI. Estimation of the mitochondrial membrane potential using fluorescence lifetime imaging microscopy. Cytometry A. 2020;97(5):471-82.

78. Morizane R, Bonventre JV. Kidney organoids: a translational journey. Trends Mol Med. 2017;23(3):246-63.

79. Stein MC, Braun F, Krebs CF, Bunders MJ. Kidney organoid systems for studies of immune-mediated kidney diseases: challenges and opportunities. Cell Tissue Res. 2021;385(2):457-73.

80. Chen WY, Evangelista EA, Yang J, Kelly EJ, Yeung CK. Kidney organoid and microphysiological kidney chip models to accelerate drug development and reduce animal testing. Front Pharmacol. 2021;12:695920.

\section{Publisher's Note}

Springer Nature remains neutral with regard to jurisdictional claims in pub-

lished maps and institutional affiliations.

Ready to submit your research? Choose BMC and benefit from:

- fast, convenient online submission

- thorough peer review by experienced researchers in your field

- rapid publication on acceptance

- support for research data, including large and complex data types

- gold Open Access which fosters wider collaboration and increased citations

- maximum visibility for your research: over $100 \mathrm{M}$ website views per year

At $\mathrm{BMC}$, research is always in progress.

Learn more biomedcentral.com/submissions 